\title{
Structural Holes in Directed Fuzzy Social Networks
}

\author{
Renjie Hu and Guangyu Zhang \\ School of Management, Guangdong University of Technology, No. 161 Yinglong Road, Tianhe District, Guangzhou 510520, China \\ Correspondence should be addressed to Guangyu Zhang; guangyu@gdut.edu.cn
}

Received 9 October 2013; Revised 30 December 2013; Accepted 31 December 2013; Published 25 February 2014

Academic Editor: Reinaldo Martinez Palhares

Copyright (C) 2014 R. Hu and G. Zhang. This is an open access article distributed under the Creative Commons Attribution License, which permits unrestricted use, distribution, and reproduction in any medium, provided the original work is properly cited.

\begin{abstract}
The structural holes have been a key issue in fuzzy social network analysis. For undirected fuzzy social networks where edges are just present or absent undirected fuzzy relation and have no more information attached, many structural holes measures have been presented, such as key fuzzy structural holes, general fuzzy structural holes, strong fuzzy structural holes, and weak fuzzy structural holes. There has been a growing need to design structural holes measures for directed fuzzy social networks, because directed fuzzy social networks where edges are attached by directed fuzzy relation would contain rich information. In this paper, we extend structural holes theory to directed fuzzy social network and propose the algorithm of unidirectional fuzzy structural holes and bidirectional fuzzy structural holes, which unveil more structural information of directed fuzzy social networks. Furthermore, we investigate the validness of the algorithm by illustrating this method to a case called G-Y Research Team and obtain reliable results, which provide strong evidences of the new measure's utility.
\end{abstract}

\section{Introduction}

A social network is a set of nodes representing people, groups, organizations, enterprises, and so forth, that are connected by links showing relations or flows between them. Social network analysis studies the implications of the restrictions of different actors in their communications and then in their opportunities of relation. The fewer constraints an actor faces, the more opportunities he/she will have, and thus he will be in a more favorable position to bargain in exchanges and to intermediate in the bargains of others that need him, increasing his influence.

It is now widely agreed that knowledge of the structure of interaction among individuals is important for a proper understanding of a number of important questions in economics, such as the spread of new ideas and technologies, the patterns of employment and wage inequality, competitive strategies in dynamic markets, and career profiles of managers [1]. Connections facilitate timely access to important information-on trade opportunities, job vacancies, network exchange, project deadlines, and novel ideas for research [2-4]. In some important instances-for example, trade opportunities - the payoffs an individual entity gets in a network will clearly depend on his relative importance in bridging gaps in the network between others. The potential benefits from bridging different parts of a network were important in the early work of Granovetter [5] and are central to the notion of structural holes developed by Burt [6]. Burt explains competitive advantage (for promotion, profit, or other kinds of success) by structural autonomous positions in a social network. He also provides a mathematical model of structural autonomy. Gnyawali et al. [7] argued that firms that have advantageous network positions-which are with a large number of connections and are therefore highly central and which are having nonredundant ties and are therefore highly structurally autonomous-gain several such benefits from their coopetitive network and become more competitively aggressive.

Once "structural holes theory" was put forth, there is a strong impact on economic sociology, organizational behavior, management, and other fields [8]. In recent years, a number of empirical studies have also shown that individuals or organizations who bridge "structural holes" in networks gain significant payoff advantages. Bruggeman et al. [9] fix Burt's model by introducing a network measure of niche overlap. Burt [10] studied the relation of structural holes and good ideas. The between-group brokers are more likely to express ideas, less likely to have ideas dismissed, and more 
likely to have ideas evaluated as valuable. Stability is one of the key issues for industrial ecosystems and industrial communities. By introducing the "structural holes" thinking in social networks theories, a weighted connectance is proposed for industrial communities. Compared with nonweighted connectance, the weighted connectance can reflect different levels of importance to different nodes and connections [11]. Rodan [12] empirically tests a mediated moderation model that distinguishes between the five different theoretical mechanisms: autonomy, competition, information brokering, opportunity recognition, and innovativeness. He suggested that of these five theoretical causal motors, innovativeness plays a key role in linking network structure and network content to performance. Sheng and Fan [13] applied the structural holes classification theory to the management of stakeholders in innovation network so as to explore preliminarily the management of stakeholders in innovation network. Zhao and Guo [14] studied the influence on innovative performance from the structural hole degree (SHD) of the network position of enterprises in the network of strategic alliances. The studies show that the SHD of a firm will play a significant role in promoting innovation performance of the firm in alliance network. Based on previous theoretical and empirical research, Haojun developed a theoretical model to explain the ways by which weak ties, structural holes, and network diversity influence firm innovation [15].

The notion of a fuzzy social network (FSN) and the methods of fuzzy social network analysis (FSNA) have attracted considerable interest and curiosity from the social and behavioral science community in recent decades. Nair and Sarasamma [16] applied fuzzy theory to perform social network analysis and defined fuzzy social network as a fuzzy graph with the entities as the nodes or actors and the relations among them as the edges or links. Liao and $\mathrm{Hu}$ [17] defined the concept of undirected fuzzy social network as a fuzzy relational structure $\widetilde{G}=(V, \widetilde{E})$, where $V$ is a nonempty set of actors or nodes and $\widetilde{E}$ is an undirected fuzzy relation on $V$. They defined some concepts of undirected fuzzy social network such as fuzzy walks and fuzzy trails and explored some of its basic properties. The definition and relevant analysis provided the theoretical foundation for further study of the undirected fuzzy social network. Fan et al. $[18,19]$ generalized the notion of regular equivalences to fuzzy social networks. They considered two kinds of generalization: regular similarity and generalized regular equivalence (GRE). Regular similarity is generalized according to the commutativity between the similarity relation and the underlying fuzzy relation, while the GRE is generalized according to the equivalence of neighborhoods of equivalent nodes. In addition, they discuss the significance of regular similarity in different applications, namely, the analysis of fuzzy state transition systems and imprecise relational data. Liao et al. [20] studied the position and role with the concepts of structural equivalence in the fuzzy technology innovation network. By means of procedures drawing on the notion of fuzzy structural equivalence from fuzzy social network analysis, 4 technological positions are clustered.
In addition, the positions in the network form a core and periphery structure. For the aspect of R\&D strategy, they suggested that decision makers should have more focus on the macroenvironment development in the core position and pay attention to the trend of other influencing factors in the periphery position. Tseng [21] proposed FNBSC (fuzzy network balanced scorecard) as a performance evaluation method when the aspects and criteria are dependent and interaction is uncertain. Liao et al. [22] extended the notion of centrality to the fuzzy framework and proposed fuzzy degree centrality, fuzzy closeness centrality and fuzzy betweenness centrality, in undirected fuzzy social network. $\mathrm{Hu}$ et al. [23] extended the notion of centrality and centralization to the fuzzy framework, proposed fuzzy inversely attenuation closeness centrality, and discussed fuzzy group closeness centralization based on inverse attenuation factor in undirected fuzzy social networks. Liao et al. [24] extended structural holes theory to undirected fuzzy social networks, studied the method of calculating the number of structural holes that actors occupy and methods for their classification, and discussed their applications in fuzzy enterprises technological innovation network. The results show that the top 5 factors are research and development capabilities, human resources quality, level of economic development, market demand, and degree of industrial clusters, and the number of their structural holes is $129,85,21,21$, and 21 , respectively. These five influence factors are the hub and mediation for the interrelation and interaction with other factors, and they promote technological innovation positively.

The fuzzy relation between actors is assumed to be undirected in undirected fuzzy social network until now. However, fuzzy relations are always directed between actors in many situations, such as fuzzy relationship between actors in directed fuzzy communication network. In directed fuzzy communication network, the claim of friendship is directed from one actor to another. Actor $i$ may choose actor $j$ as a friend, but that does not necessarily imply actor $j$ chooses actor $i$ as a friend. Considering the directed fuzzy relation, we define the concept of directed fuzzy social networks and develope some concepts such as fuzzy directed walk, fuzzy directed trail, fuzzy directed path, and fuzzy semipath for directed fuzzy social network. Using the concepts of fuzzy path and fuzzy semipath, we define fuzzy connectivity of pairs of actors and the fuzzy connectedness of a directed fuzzy social network. In this paper, we extend structural holes theory to directed fuzzy social network based on directed fuzzy relation and propose the algorithm of unidirectional fuzzy structural holes and bidirectional fuzzy structural holes by applying the concept of connectedness to directed fuzzy social network.

The organization of this paper is as follows. Section 2 contains the notation and some preliminary concepts. In Section 3, we propose four kinds of connectivity in directed fuzzy social network. In Section 4, the algorithm of unidirectional fuzzy structural holes and bidirectional fuzzy structural holes is described. Analytical and numerical results based on various structural holes measures applying to $G-Y$ Research Team are shown in Section 5. Finally a conclusion appears in Section 6. 


\section{Preliminaries}

Many social network measures have been defined for binary situations in which a pair of actors is either connected or not. However, the relation between actors is generally characterized by vague notions, such as "equally," "moderately," "strongly," "very strongly," "extremely," and a "significant degree." The fuzzy set theory, introduced by Zadeh $[25,26]$, is suitable for dealing with the uncertainty and imprecision associated with information concerning various parameters. Liao and $\mathrm{Hu}$ [17] applied fuzzy theory to social network and defined undirected fuzzy social network as follows.

Definition 1. An undirected fuzzy social network is defined as a fuzzy relational structure $\widetilde{G}=(V, \widetilde{E})$, where $V=$ $\left\{v_{1}, v_{2}, \ldots, v_{n}\right\}$ is a nonempty set of actors or nodes and $\widetilde{E}=$ $\left(\begin{array}{ccc}\widetilde{e_{11}} & \cdots & \widetilde{e_{1 n}} \\ \vdots & \ddots & \vdots \\ \widetilde{e_{n 1}} & \cdots & \overline{\vec{e}}_{n n}\end{array}\right)$ is an undirected fuzzy relation on $V$.

Many fuzzy relations are directional. A fuzzy relation is directional if the ties are oriented from one actor to another. So, we define directed fuzzy social network (DFSN) as follows.

Definition 2. A directed fuzzy social network is defined as a fuzzy relational structure $\widetilde{G}_{d n}=\left(V, \widetilde{E}_{d n}\right)$, where $V=$ $\left\{v_{1}, v_{2}, \ldots, v_{n}\right\}$ is a nonempty set of actors or nodes and $\widetilde{E}_{d n}=$ $\left(\begin{array}{ccc}\widetilde{e_{11}} & \ldots & \widetilde{e_{1 n}} \\ \vdots & \ddots & \vdots \\ \widetilde{e_{n 1}} & \cdots & \frac{\vec{e}_{n n}}{n_{n n}}\end{array}\right)$ is a directed fuzzy relation on $V$.

Fuzzy social network includes undirected fuzzy social network and directed fuzzy social network. The major difference between directed fuzzy social network and undirected fuzzy social network lies in the fact that directed fuzzy relation is taken into consideration. According to Definition 1, $\widetilde{e_{i j}}$ is equal to $\widetilde{e_{j i}}$ in undirected fuzzy social network. However, $\widetilde{e_{i j}}$ is not always equal to $\widetilde{e_{j i}}$ in directed fuzzy social network. In Definition 2, $\widetilde{E}_{d n}$ is called directed fuzzy adjacency matrix of $\widetilde{G}_{d n} \cdot \mu_{\widetilde{E}_{d n}}=\left(\begin{array}{ccc}\mu\left(\widetilde{e_{11}}\right) & \cdots & \mu\left(\widetilde{e_{1 n}}\right) \\ \vdots & \ddots & \vdots \\ \mu\left(\widetilde{\overrightarrow{e n}_{n 1}}\right) & \cdots & \mu\left(\widetilde{\bar{e}_{n n}}\right)\end{array}\right)$ is a membership function of $\widetilde{E}_{d n}$, which is asymmetrical matrix.

One of the most important subnetworks in a directed fuzzy social network is the directed fuzzy ternary social network, consisting of three actors and the fuzzy relations between them. A directed fuzzy ternary social network can be presented as a fuzzy relational structure $\widetilde{G}_{d 3}=\left(V, \widetilde{E}_{d 3}\right)$, where $V=\left\{v_{1}, v_{2}, v_{3}\right\}$ is a set of three actors and $\widetilde{E}_{d 3}=\left(\begin{array}{ccc}\overline{\bar{e}_{11}} & \widetilde{e_{12}} & \overline{e_{13}} \\ \overline{e_{21}} & \frac{e_{22}}{e_{32}} & e_{23} \\ e_{33}\end{array}\right)$ is a directed fuzzy relation on $V$.

The related concepts of undirected fuzzy social networks can also be used in a directed fuzzy social network, but the direction of the fuzzy relation between actors must be taken into account.

We now discuss how several of the concepts for social networks are applied to directed fuzzy social networks.

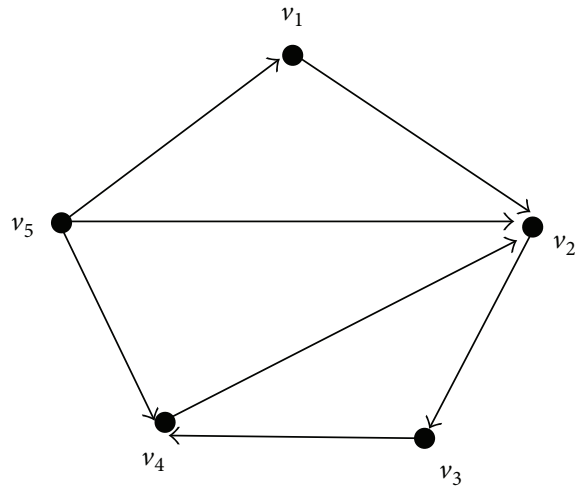

Figure 1: A directed fuzzy communication network of five actors.

We will focus on the most important directed fuzzy social network concepts including the fuzzy directed walk, fuzzy directed trail, fuzzy directed path, and fuzzy semipath.

Definition 3. A fuzzy directed walk is a sequence of alternating actors and fuzzy relation so that each fuzzy relation has its origin at the previous actor and its terminus at the subsequent actors.

More simply, in a directed fuzzy walk, all fuzzy relations are "pointing" in the same direction.

Definition 4. A fuzzy directed trail in a directed fuzzy social network is a fuzzy directed walk in which no fuzzy relation is included more than once.

Definition 5. A fuzzy directed path in a directed fuzzy social network is a fuzzy directed walk in which no actor and no fuzzy relation is included more than once.

Definition 6. A fuzzy semipath joining actors $v_{i}$ and $v_{j}$ is a sequence of distinct actors, where all successive pairs of actors are connected by a fuzzy relation from the first to the second or by a fuzzy relation from the second to the first for all successive pairs of actors.

In a fuzzy semipath, the direction of the fuzzy relations is irrelevant. Note that every fuzzy path is a fuzzy semipath, but not every fuzzy semipath is a fuzzy path.

Example 7. Consider the directed fuzzy communication network $\widetilde{G}_{d 5}=\left(V, \widetilde{E}_{d 5}\right)$ (see Figure 1) with $V=\left\{v_{1}, v_{2}, v_{3}, v_{4}, v_{5}\right\}$ and $\mu_{\widetilde{E}_{d 5}}=\left(\begin{array}{ccccc}1 & 0.2 & 0 & 0 & 0 \\ 0 & 1 & 0.5 & 0 & 0 \\ 0 & 0 & 1 & 0.7 & 0 \\ 0 & 0.4 & 0 & 1 \\ 0.9 & 0.2 & 0 & 0.8 & 0\end{array}\right)$, which is an asymmetrical matrix, is a membership function of $\widetilde{E}_{d 5}$.

In Figure $1, \widetilde{W}=v_{5} v_{1} v_{2} v_{3} v_{4} v_{2} v_{3}$ is a fuzzy directed walk, $\widetilde{T}=v_{5} v_{4} v_{2} v_{3} v_{4}$ fuzzy directed trail, $\widetilde{P}=v_{5} v_{1} v_{2} v_{3} v_{4}$ fuzzy directed path, and $\widetilde{S P}=v_{1} v_{5} v_{4} v_{3} v_{2}$ a fuzzy semipath. 


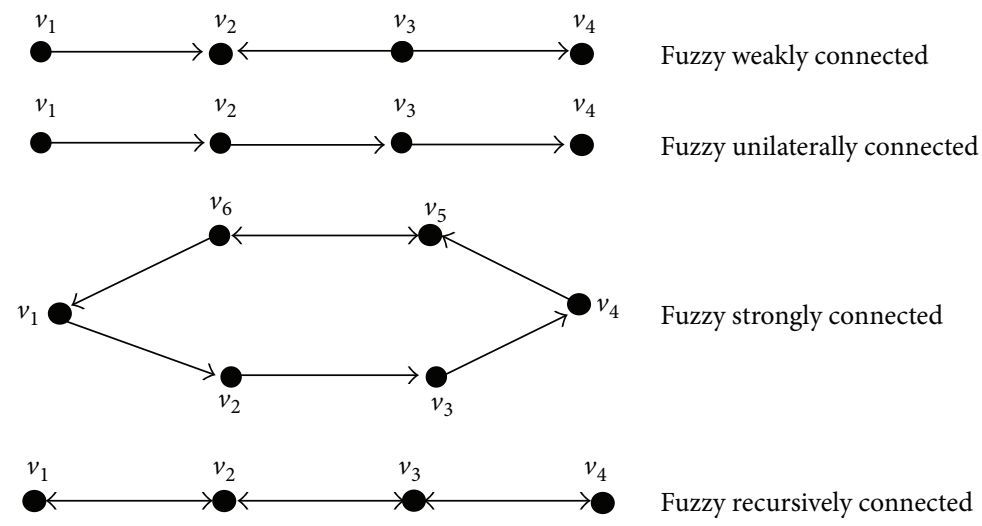

FIGURE 2: Different kinds of connectivity in a directed fuzzy social network.

\section{Connectivity in Directed Fuzzy Social Networks}

Using the ideas of fuzzy path and fuzzy semipath, we can now define connectivity of pairs of actors and the connectedness of a directed fuzzy social network.

3.1. Pairs of Actors. In an undirected fuzzy social network a pair of actors is reachable if there is a fuzzy path between them. However, in order to define reachability in a directed fuzzy social network, we must consider fuzzy directed paths. Specifically, if there is a fuzzy directed path from $v_{i}$ to $v_{j}$, then $v_{j}$ is reachable from $v_{i}$.

Consider now both fuzzy directed paths and fuzzy semipaths between pairs of actors. We can define four different ways that two actors can be connected by some fuzzy directed paths or fuzzy semipaths. A pair of actors, $v_{i}, v_{j}$, is

(1) fuzzy weakly connected if they are joined by a fuzzy semipath,

(2) fuzzy unilaterally connected if they are joined by a fuzzy directed path from $v_{i}$ to $v_{j}$, or a fuzzy directed path from $v_{j}$ to $v_{i}$,

(3) fuzzy strongly connected if there is a fuzzy directed path from $v_{i}$ to $v_{j}$ and a fuzzy directed path from $v_{j}$ to $v_{i}$; the path from $v_{i}$ to $v_{j}$ may contain different actors and fuzzy relations than the path from $v_{j}$ to $v_{i}$,

(4) fuzzy recursively connected if they are fuzzy strongly connected, and the path from $v_{i}$ to $v_{j}$ uses the same actors and fuzzy relations as the path from $v_{j}$ to $v_{i}$, in reverse order.

Notice that these forms of connectivity are increasingly strict and that any strict form implies connectivity of any less strict form. For example, any two actors that are fuzzy recursively connected are also fuzzy strongly connected, fuzzy unilaterally connected, and fuzzy weakly connected. Figure 2 illustrates these different kinds of connectivity.

3.2. Connectedness of Directed Fuzzy Social Networks. It is now possible to define four different kinds of connectivity for directed fuzzy social network. If a directed fuzzy social network is connected, then it is connected by one of these four kinds of connectivity; otherwise, it is not connected. Since there are four types of connectivity between pairs of actors in a directed fuzzy social network, there are four definitions of connectivity for a directed fuzzy social network. A directed fuzzy social network is

(1) fuzzy weakly connected if all pairs of actors are fuzzy weakly connected,

(2) fuzzy unilaterally connected if all pairs of actors are fuzzy unilaterally connected,

(3) fuzzy strongly connected if all pairs of actors are fuzzy strongly connected,

(4) fuzzy recursively connected if all pairs of actors are fuzzy recursively connected.

In a fuzzy weakly connected directed fuzzy social network, all pairs of actors are connected by a fuzzy semipath. In a fuzzy unilaterally connected directed fuzzy social network, between each pair of actors there is a fuzzy directed path from one actor to the other; in other words, at least one actor is reachable from the other in the pair. In a fuzzy strongly connected directed fuzzy social network, each actor in each pair is reachable from the other; there is a fuzzy directed path from each actor to each other actor. In a fuzzy recursively connected directed fuzzy social network, each actor, in each pair, is reachable from the other, and the fuzzy directed paths contain the same actors and fuzzy relations but in reverse order. As with the definitions of connectivity for pairs of actors, these are increasingly strict connectivity definitions.

From these definitions, it should be clear that every fuzzy strongly connected directed fuzzy social network is fuzzy unilaterally connected, but the reverse is not true.

\section{The Measures of Structural Holes of Directed Fuzzy Social Networks}

Connectivity of directed fuzzy social network is the basis of the study of its structural holes. According to the characteristics of connectivity of directed fuzzy social network, 


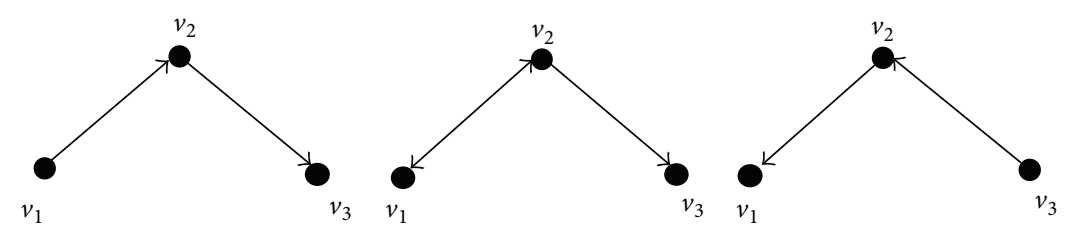

(a)

(b)

(c)

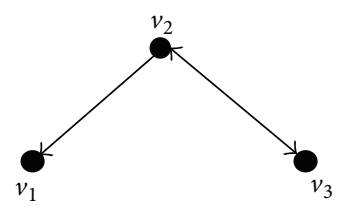

(d)

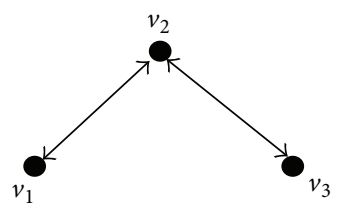

(e)

Figure 3: Structural holes of directed fuzzy ternary social network.

the analysis of their structural holes and its algorithm are as follows.

4.1. Structural Holes of Directed Fuzzy Ternary Social Networks. According to the features of connectivity of directed fuzzy ternary social network, $\widetilde{G}_{d 3}=\left(V, \widetilde{E}_{d 3}\right)$, the structural holes in $\widetilde{G}_{d 3}=\left(V, \widetilde{E}_{d 3}\right)$ can be divided into five types as follows.

(1) In the directed fuzzy ternary social network $\widetilde{G}_{d 3}=$ $\left(V, \widetilde{E}_{d 3}\right), \widetilde{e}_{12}>0, \widetilde{e}_{23}>0$, and the other elements in $\widetilde{E}_{d 3}$ are equal to 0 (see Figure $3(\mathrm{a})$ ).

(2) In the directed fuzzy ternary social network $\widetilde{G}_{d 3}=$ $\left(V, \widetilde{E}_{d 3}\right), \widetilde{e}_{12}>0, \widetilde{e}_{21}>0, \widetilde{e}_{23}>0$, and the other elements in $\widetilde{E}_{d 3}$ are equal to 0 (see Figure 3(b)).

(3) In the directed fuzzy ternary social network $\widetilde{G}_{d 3}=$ $\left(V, \widetilde{E}_{d 3}\right), \widetilde{e}_{21}>0, \widetilde{e}_{32}>0$, and the other elements in $\widetilde{E}_{d 3}$ are equal to 0 (see Figure 3(c)).

(4) In the directed fuzzy ternary social network $\widetilde{G}_{d 3}=$ $\left(V, \widetilde{E}_{d 3}\right), \widetilde{e}_{21}>0, \widetilde{e}_{32}>0, \widetilde{e}_{23}>0$, and the other elements in $\widetilde{E}_{d 3}$ are equal to 0 (see Figure $3(\mathrm{~d})$ ).

(5) In the directed fuzzy ternary social network $\widetilde{G}_{d 3}=$ $\left(V, \widetilde{E}_{d 3}\right), \widetilde{e}_{12}>0, \widetilde{e}_{21}>0, \widetilde{e}_{23}>0, \widetilde{e}_{32}>0$, and the other elements in $\widetilde{E}_{d 3}$ are equal to 0 (see Figure $3(\mathrm{e})$ ).

There are five types of structural holes in the directed fuzzy ternary social network $\widetilde{G}_{d 3}=\left(V, \widetilde{E}_{d 3}\right)$ (see Figure 3). The structural holes that $v_{2}$ occupies in Figures 3(a), 3(b), $3(\mathrm{c})$, and 3(d) are unidirectional fuzzy structural holes, and that which $v_{2}$ occupies in Figure 3(e) is a bidirectional fuzzy structural hole.

4.2. Structural Holes and Their Algorithm of Directed Fuzzy Social Networks. On the basis of the fuzzy unilateral connectedness and fuzzy recursive connectedness of directed fuzzy social network, we define unidirectional fuzzy structural holes and bidirectional fuzzy structural holes as follows.

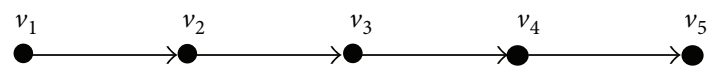

(a)

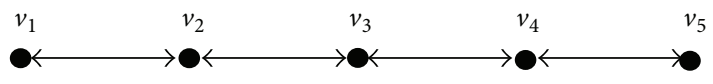

(b)

Figure 4: Two directed fuzzy social networks.

Definition 8. In a directed fuzzy social network $\widetilde{G}_{d n}=$ $\left(V, \widetilde{E}_{d n}\right)$, if there is fuzzy unilaterally connected from $v_{i}$ to $v_{j}$, and $v_{k}$ is on the path from $v_{i}$ to $v_{j}$, then $v_{k}$ is called to occupy a unidirectional fuzzy structural hole from $v_{i}$ to $v_{j}$.

In Definition $8, k \neq i \neq j$. If $v_{k}$ occupies a unidirectional fuzzy structural hole from $v_{i}$ to $v_{j}$, it means that $v_{i}$ to $v_{j}$ is not reachable without $v_{k}$. The actor $v_{k}$ can get to information interests or control interests from $v_{i}$ to $v_{j}$.

Definition 9. In a directed fuzzy social network $\widetilde{G}_{d n}=$ $\left(V, \widetilde{E}_{d n}\right)$, if there is fuzzy recursively connected from $v_{i}$ to $v_{j}$, and $v_{k}$ is on the path between $v_{i}$ and $v_{j}$, then $v_{k}$ is called to occupy a bidirectional fuzzy structural hole between $v_{i}$ and $v_{j}$.

In Definition $9, k \neq i \neq j$. If $v_{k}$ occupies a bidirectional fuzzy structural hole between $v_{i}$ and $v_{j}$, it means that $v_{i}$ and $v_{j}$ are not reachable without $v_{k}$. The actor $v_{k}$ can get to information interests or control interests between $v_{i}$ and $v_{j}$.

For example, in Figure 4(a), $v_{2}$ occupies a unidirectional fuzzy structural hole from $v_{1}$ to $v_{3}$, a unidirectional fuzzy structural hole from $v_{1}$ to $v_{4}$, and a unidirectional fuzzy structural hole from $v_{1}$ to $v_{5}$. The actor $v_{3}$ occupies a unidirectional fuzzy structural hole from $v_{1}$ to $v_{4}$, a unidirectional fuzzy structural hole from $v_{1}$ to $v_{5}$, and a unidirectional fuzzy structural hole from $v_{2}$ to $v_{4}$, a unidirectional fuzzy structural hole from $v_{2}$ to $v_{5}$. In Figure $4(\mathrm{~b}), v_{2}$ occupies a bidirectional fuzzy structural hole between $v_{1}$ and $v_{3}$, and a bidirectional fuzzy structural hole between $v_{1}$ and $v_{4}$, a bidirectional fuzzy 
structural hole between $v_{1}$ and $v_{5}$. The actor $v_{3}$ occupies a bidirectional fuzzy structural hole between $v_{1}$ and $v_{4}$, a bidirectional fuzzy structural hole between $v_{1}$ and $v_{5}$, and a bidirectional fuzzy structural hole between $v_{2}$ and $v_{4}$, a bidirectional fuzzy structural hole between $v_{2}$ and $v_{5}$.

The relationships between actors in a directed fuzzy social network are directional but are not links between any two actors. Hence if there is opportunity to establish contact with the actors with whom there is no fuzzy relation and get access to interests from information or control, the actors in directed fuzzy social networks will endeavor to seek such positions to build their unidirectional or bidirectional structural holes. In a specific directed fuzzy social network, how to find out unidirectional and bidirectional fuzzy structural holes, how to calculate their unidirectional and bidirectional fuzzy structural holes that actors occupy, and how to determine the influence that unidirectional and bidirectional fuzzy structural holes make on the connectedness degree of directed fuzzy social networks, thereby discriminating the importance of unidirectional and bidirectional fuzzy structural holes, are precisely the issues to be studied in this paper.

\subsubsection{Algorithm for Unidirectional Fuzzy Structural Holes}

Step 1. In a directed fuzzy social network $\widetilde{G}_{d n}=\left(V, \widetilde{E}_{d n}\right)$, seek the fuzzy directed paths $\widetilde{\omega}_{i},(i=1,2, \ldots, m)$, which is through $v_{k}$, and the actors are not less than 3 .

Step 2. Calculate the number of actors on the right and left of actor $v_{k}$ on any fuzzy directed paths $\widetilde{\omega}_{i}$. (If $v_{k}$ is the starting point, then there is no actor on the left, so $v_{k}$ occupies 0 unidirectional fuzzy structural holes. If $v_{k}$ is the terminal point, then there is no actors on the right of $v_{k}$, so $v_{k}$ occupies 0 unidirectional fuzzy structural holes.)

Step 3. Calculate the numbers of unidirectional fuzzy structural holes that $v_{k}$ occupies on any fuzzy directed paths $\widetilde{\omega}_{i}$. If on the fuzzy directed paths $\widetilde{\omega}_{i}$, there are $p_{i}$ actors on the left of $v_{k}$ and $q_{i}$ actors on the right, then the numbers of unidirectional fuzzy structural holes $v_{k}$ occupies are $p_{i} \cdot q_{i}$.

Step 4. According to the calculated result in Step 3, the numbers of unidirectional fuzzy structural holes that $v_{k}$ occupies are fus $\left(v_{k}\right)=\left(\sum_{i=1}^{m} p_{i} \cdot q_{i}\right)-u$, where $u$ is the repetitive computation numbers of unidirectional fuzzy structural holes on the fuzzy directed path $\widetilde{\omega}_{i}$.

\subsubsection{Algorithm for Bidirectional Fuzzy Structural Holes}

Step 1. In a directed fuzzy social network $\widetilde{G}_{d n}=\left(V, \widetilde{E}_{d n}\right)$, seek the fuzzy recursively connected paths $\widetilde{\lambda}_{i},(i=1,2, \ldots, m)$, which is through $v_{k}$, and the actors are not less than 3.

Step 2. Calculate the number of actors on the right and left of actor $v_{k}$ on any fuzzy recursively connected paths $\tilde{\lambda}_{i}$. (If $v_{k}$ is the starting point, then there is no actor on the left, so $v_{k}$ occupies 0 bidirectional fuzzy structural holes. If $v_{k}$ is the terminal point, then there are no actors on the right of $v_{k}$, so $v_{k}$ occupies 0 bidirectional fuzzy structural holes.)

Step 3. Calculate the numbers of bidirectional fuzzy structural holes that $v_{k}$ occupies on any fuzzy recursively connected paths $\tilde{\lambda}_{i}$. If on the fuzzy recursively connected paths $\tilde{\lambda}_{i}$, there are $p_{i}$ actors on the left of $v_{k}$ and $q_{i}$ actors on the right, then the numbers of bidirectional fuzzy structural holes $v_{k}$ occupies are $p_{i} \cdot q_{i}$.

Step 4. According to the calculated result in Step 3, the numbers of bidirectional fuzzy structural holes that $v_{k}$ occupies are $\operatorname{fbs}\left(v_{k}\right)=\left(\sum_{i=1}^{m} a_{i} \cdot b_{i}\right)-v$, where $v$ is the repetitive computation numbers of bidirectional fuzzy structural holes on the fuzzy recursively connected path $\tilde{\lambda}_{i}$.

According to the algorithms above, the numbers of unidirectional and bidirectional fuzzy structural holes that any actors occupies in a directed fuzzy social networks can be calculated, and thus the influence on the connectedness degree of directed fuzzy social networks from actors can be determined.

\section{Case Study}

$\widetilde{G}_{d 8}=\left(V, \widetilde{E}_{d 8}\right)$ is a directed fuzzy communication network of the G-Y Research Team, $V=\left\{v_{1}, v_{2}, \ldots, v_{8}\right\}$ is a set of 8 researchers, and $\widetilde{E}_{d 8}$ is directed fuzzy communication relation on $V$. We got the fuzzy communication relations among 8 researchers (see (1)) of G-Y Research Team by investigation.

Consider

$$
\mu_{\widetilde{E}_{d 8}}=\left(\begin{array}{cccccccc}
1 & 0.3 & 0 & 0.8 & 0 & 0 & 0 & 0 \\
0 & 1 & 0 & 0 & 0 & 0 & 0 & 0 \\
0 & 0 & 1 & 0 & 0.6 & 0.9 & 0 & 0 \\
0 & 0 & 0 & 1 & 0 & 0.4 & 0.2 & 0 \\
0 & 0.1 & 0 & 0 & 1 & 0.7 & 0 & 0 \\
0 & 0 & 0 & 0.5 & 0.7 & 1 & 0 & 0 \\
0 & 0 & 0 & 0 & 0 & 0 & 1 & 0 \\
0 & 0 & 0 & 0 & 0.2 & 0 & 0 & 1
\end{array}\right) .
$$

We used Netdraw in UCINET to draw the directed fuzzy communication network of the G-Y Research Team (ties have values $>0$ ) (see Figure 5).

According to the algorithms above, the numbers of unidirectional and bidirectional fuzzy structural holes of each researcher were calculated (see Table 1).

It shows that only $v_{6}$ occupies a bidirectional fuzzy structural hole in the G-Y Research Team. It means that $v_{6}$ can get more information benefits and control benefits than the other seven researchers. Table 1 shows the top three researchers who occupy the number of unidirectional fuzzy structural holes are $v_{4}, v_{5}$ and $v_{6}$, respectively 7,7 and 5 . They are the hub and intermediaries for other researchers, which play a positive role in promoting the communication of the G-Y Research Team. 
TABLE 1: Numbers of fuzzy structural holes of the researchers about the G-Y Research Team.

\begin{tabular}{lccc}
\hline Researchers & $\begin{array}{c}\text { Number of } \\
\text { unidirectional } \\
\text { fuzzy structural } \\
\text { holes }\end{array}$ & $\begin{array}{c}\text { Number of } \\
\text { bidirectional } \\
\text { fuzzy structural } \\
\text { holes }\end{array}$ & Sum \\
\hline$v_{1}$ & 0 & 0 & 0 \\
$v_{2}$ & 0 & 0 & 0 \\
$v_{3}$ & 0 & 0 & 0 \\
$v_{4}$ & 7 & 0 & 7 \\
$v_{5}$ & 7 & 0 & 7 \\
$v_{6}$ & 5 & 1 & 6 \\
$v_{7}$ & 0 & 0 & 0 \\
$v_{8}$ & 0 & 0 & 0 \\
\hline
\end{tabular}

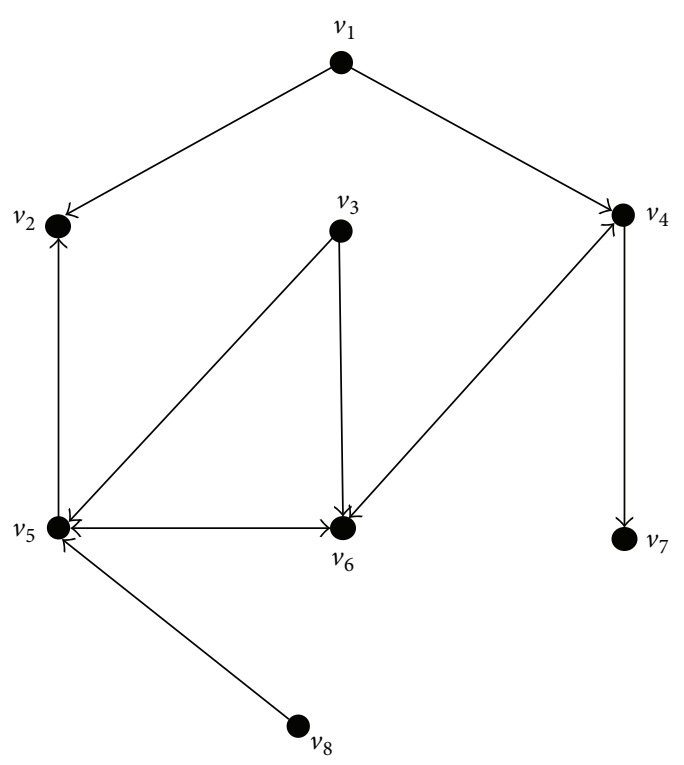

FIGURE 5: Directed fuzzy communication network of the $G-Y$ Research Team.

\section{Conclusions}

In this paper, we propose directed fuzzy social network based on directed fuzzy relation between actors. The basic concepts of directed fuzzy social network are defined and some of its basic properties are explored in this note.

Firstly, we extend the notion of connectivity to the directed fuzzy social network. There are four types of connectivity in directed fuzzy social networks: fuzzy weakly connected, fuzzy unilaterally connected, fuzzy strong connected, and fuzzy recursively connected.

Secondly, the algorithms of structural holes are discussed in this paper. There are two types of fuzzy structural holes in directed fuzzy social networks: unidirectional fuzzy structural holes and bidirectional fuzzy structural holes. The algorithms of unidirectional and bidirectional fuzzy structural holes that the actors occupy are put forward, which will provide a theoretical basis for the subsequent application study.

Thirdly, we apply fuzzy structural holes theory to the case which is called G-Y Research Team. It shows that only $v_{6}$ occupies a bidirectional fuzzy structural hole in the G-Y Research Team. It means that $v_{6}$ can get more information benefits and control benefits than the other seven researchers. The top three researchers who occupy the number of unidirectional fuzzy structural holes are $v_{5}, v_{4}$ and $v_{6}$. They are the hub and intermediaries for other researchers, playing a positive role in promoting the communication of the G-Y Research Team.

Fuzzy structural holes analysis is one of the most important and a commonly used tool in directed fuzzy social network. This study gives further supplement to the structural holes theory and provides the theoretical foundation for further study of the directed fuzzy social network.

\section{Conflict of Interests}

Renjie $\mathrm{Hu}$ and Guangyu Zhang declare that there is no conflict of interests regarding the publication of this paper.

\section{Acknowledgments}

The authors wish to give many thanks to editor and anonymous referees for their valuable feedback on earlier versions of this paper. They also acknowledge the support by the National Natural Science Foundation of China (no. 71173051), Eleventh Five-Year Plan Project of Philosophy and Social Sciences, Guangdong Province (no. GD10CGL07), and Hightech Zone development guide special projects of Guangdong Province (no. 2010A011200020).

\section{References}

[1] S. Goyal and F. Vega-Redondo, "Structural holes in social networks," Journal of Economic Theory, vol. 137, no. 1, pp. 460492, 2007.

[2] M. Granovetter, Getting a Job, Harvard University Press, Cambridge, Mass, USA, 1974.

[3] S. Cook Karen and M. Emerson Richard, "Power, equity and commitment in exchange networks," American Sociological Review, vol. 43, no. 5, pp. 721-739, 1978.

[4] R. S. Burt, "Autonomy in a social topology", American Journal of Sociology, vol. 85, no. 4, pp. 892-925, 1980.

[5] M. Granovetter, “The strength of weak ties," American Journal of Sociology, vol. 78, no. 6, pp. 1360-1380, 1974.

[6] R. S. Burt, Structural Holes: The Social Structure of Competition, Harvard University Press, Cambridge, Mass, USA, 1992.

[7] D. R. Gnyawali, J. He, and R. Madhavan, "Impact of co-opetition on firm competitive behavior: an empirical examination," Journal of Management, vol. 32, no. 4, pp. 507-530, 2006.

[8] T. M. Everet, "Structure hole review," European Sociological Review, vol. 10, no. 1, pp. 98-99, 1994.

[9] J. Bruggeman, G. Carnabuci, and I. Vermeulen, "A note on structural holes theory and niche overlap," Social Networks, vol. 25, no. 1, pp. 97-101, 2003.

[10] R. S. Burt, "Structural holes and good ideas," American Journal of Sociology, vol. 110, no. 2, pp. 349-399, 2004. 
[11] Z. Wang, L. Shi, and X. Jia, "Weighted connectance for industrial communities based on structural holes theory," Acta Ecologica Sinica, vol. 29, no. 2, pp. 810-814, 2009.

[12] S. Rodan, "Structural holes and managerial performance: Identifying the underlying mechanisms," Social Networks, vol. 32, no. 3, pp. 168-179, 2010.

[13] Y. Sheng and D. Fan, "Structural holes classification theory and its application in innovation network," Studies in Science of Science, vol. 27, no. 9, pp. 1407-1411, 2009.

[14] Y. Zhao and X. Guo, "Structural hole degree, inter-firm network and innovation performance evidence from Chinese domestic audio video industry strategic alliance," Science \& Technology Progress and Policy, vol. 29, no. 17, pp. 76-81, 2012.

[15] H. Zhou, The Search-Transfer Problem: The Curvilinear Effect of Weak Ties, Structural Holes and Network Diversity on Firm Innovation, School of Management of Zhejiang University, 2011.

[16] P. S. Nair and S. T. Sarasamma, "Data mining through fuzzy social network analysis," in Proceedings of the 26th Annual Meeting of the North American Fuzzy Information Processing Society (NAFIPS '07), pp. 251-255, June 2007.

[17] L. Liao and R. Hu, "On the definition and property analysis of fuzzy social network based on fuzzy graph," Journal of Guangdong University of Technology (Social Science Edition), vol. 12, no. 3, pp. 46-51, 2012.

[18] T.-G. Fan, C.-J. Liau, and T.-Y. Lin, "Positional analysis in fuzzy social networks," in Proceedings of the IEEE International Conference on Granular Computing (GrC '07), pp. 423-428, November 2007.

[19] T.-F. Fan, C.-J. Liau, and T.-Y. Lin, "A theoretical investigation of regular equivalences for fuzzy graphs," International Journal of Approximate Reasoning, vol. 49, no. 3, pp. 678-688, 2008.

[20] L. Liao, R. Hu, and G. Zhang, "The position analysis of the fuzzy technology innovation network," Journal of High Technology Management Research, vol. 23, no. 2, pp. 83-89, 2012.

[21] M.-L. Tseng, "Implementation and performance evaluation using the fuzzy network balanced scorecard," Computers and Education, vol. 55, no. 1, pp. 188-201, 2010.

[22] L. Liao, R. Hu, and G. Zhang, "The centrality analysis of fuzzy social networks," Fuzzy Systems and Mathematics, vol. 27, no. 2, pp. 169-173, 2012.

[23] R. Hu, G. Zhang, and L. Liao, "The closeness centrality analysis of fuzzy social network based on inversely attenuation factor," Fuzzy Information \& Engineering and Operations Research \& Management, Advances in Intelligent Systems and Computing, vol. 211, pp. 457-465, 2013.

[24] L. Liao, R. Hu, and G. Zhang, "Structural holes analysis of fuzzy social network," Journal of Southeast University (Natural Science Edition), vol. 43, no. 4, pp. 900-904, 2013.

[25] L. A. Zadeh, "Fuzzy sets," Information and Computation, vol. 8, no. 3, pp. 338-353, 1965.

[26] L. A. Zadeh, "A fuzzy-algorithmic approach to the definition of complex or imprecise concepts," International Journal of ManMachine Studies, vol. 8, no. 3, pp. 249-291, 1976. 


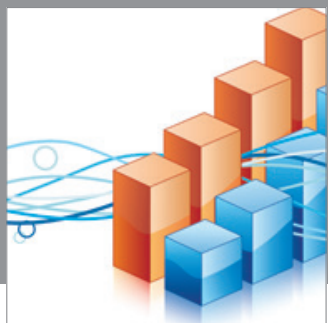

Advances in

Operations Research

mansans

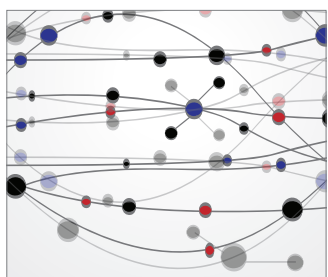

The Scientific World Journal
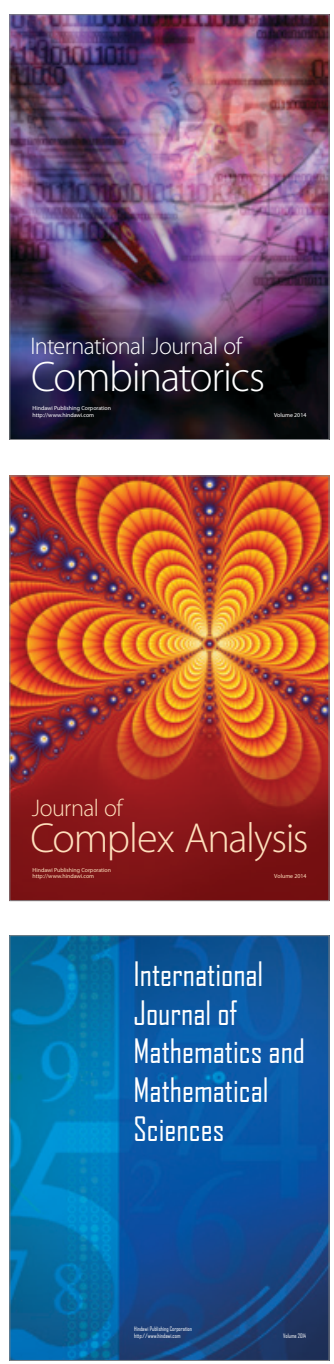
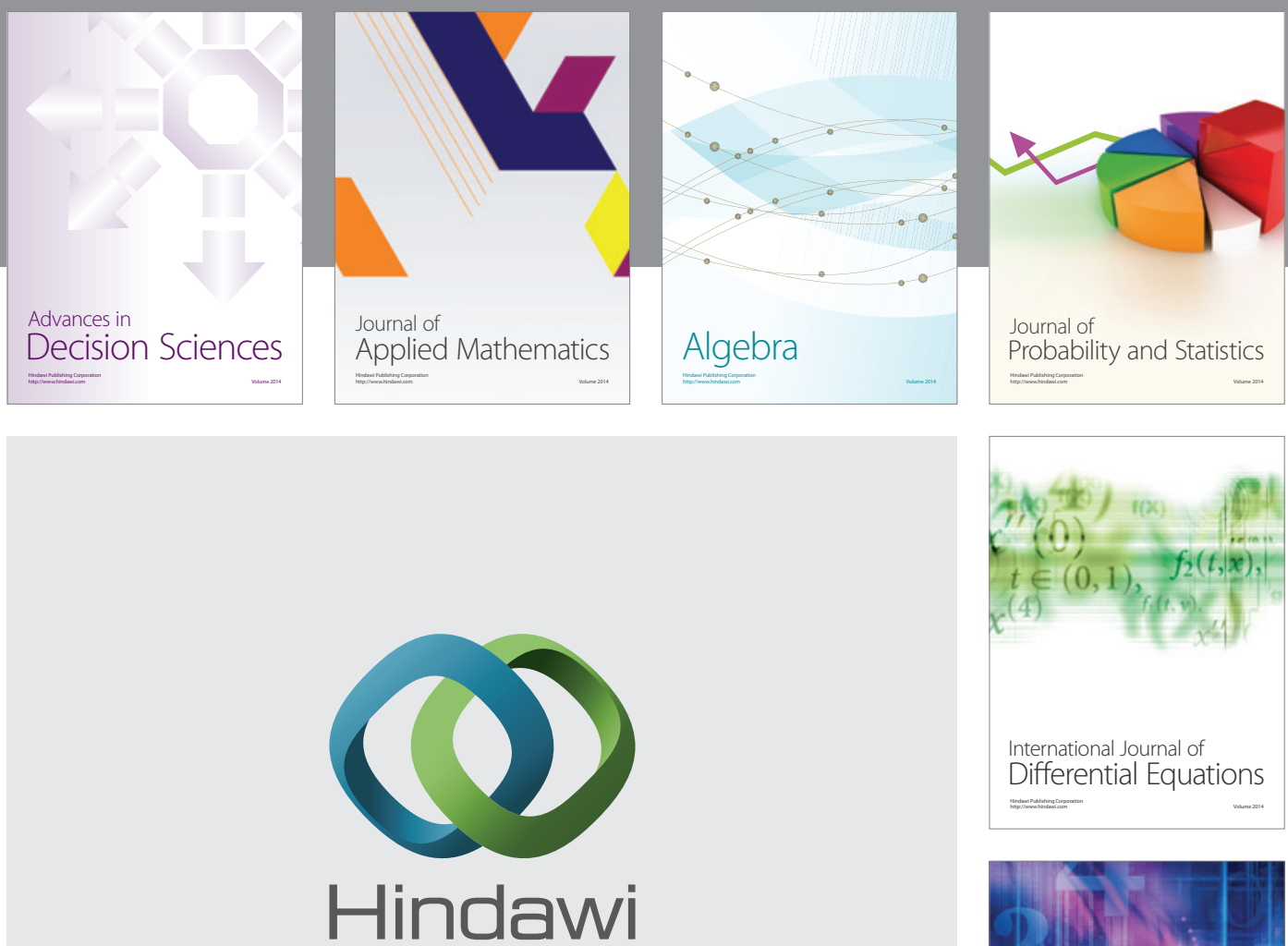

Submit your manuscripts at http://www.hindawi.com
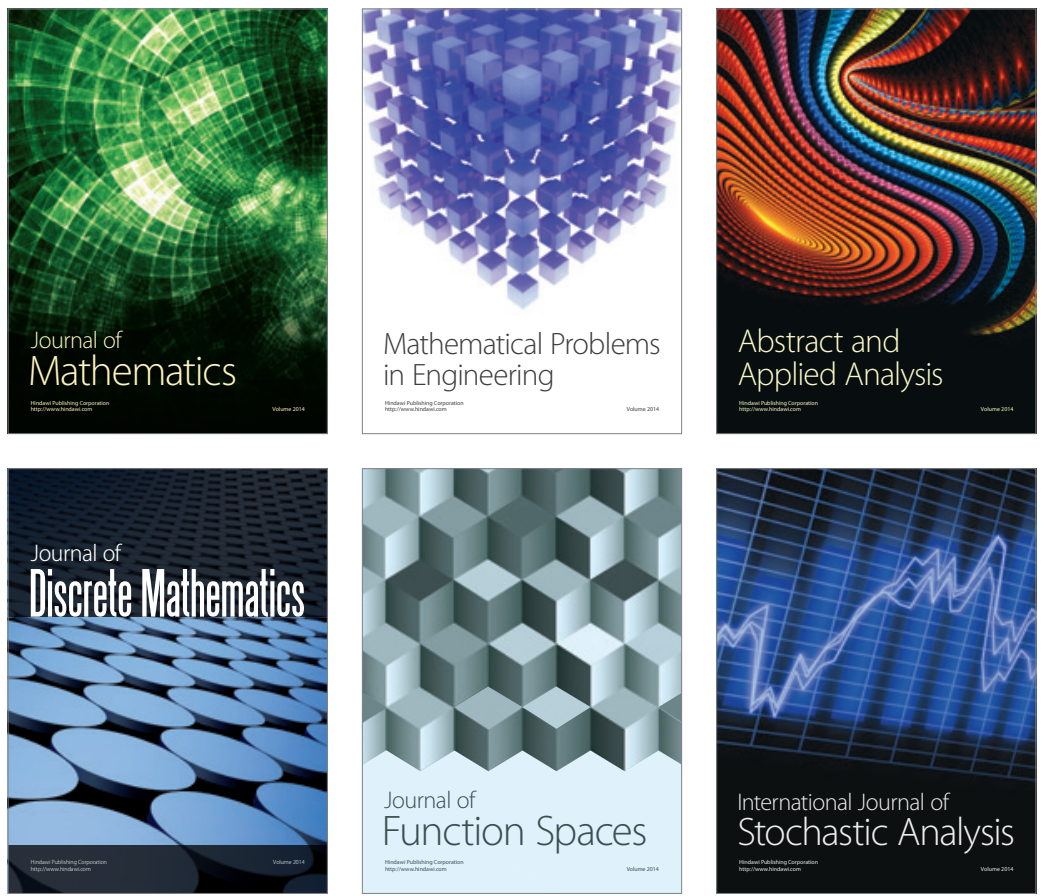

Journal of

Function Spaces

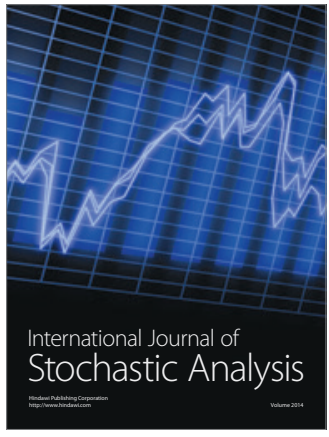

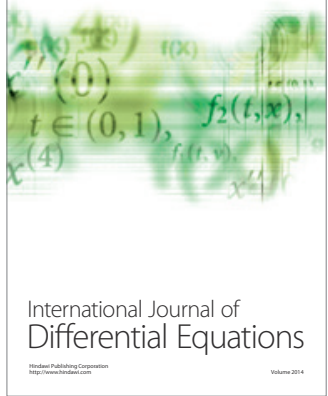
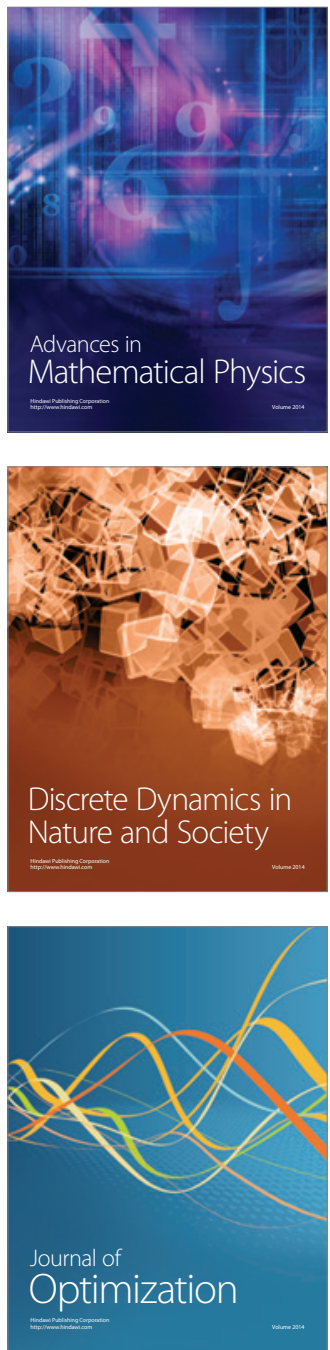